\title{
CDK2 and CDK4 play important roles in promoting the proliferation of SKOV3 ovarian carcinoma cells induced by tumor-associated macrophages
}

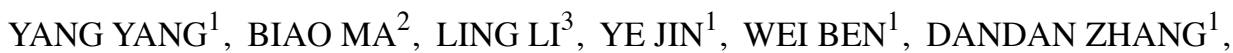 \\ KEPING JIANG ${ }^{1}$, SHUJUN FENG ${ }^{1}$, LU HUANG $^{1}$ and JIANHUA ZHENG ${ }^{1}$ \\ Departments of ${ }^{1}$ Obstetrics and Gynecology, and ${ }^{2}$ General Surgery, The First Affiliated Hospital of Harbin \\ Medical University, Harbin, Heilongjiang 150001, P.R. China; ${ }^{3}$ School of Computing, \\ University of Kent, Canterbury, Kent, CT2 7NF, UK
}

Received January 16, 2014; Accepted March 13, 2014

DOI: $10.3892 /$ or.2014.3127

\begin{abstract}
A large quantity of M2-polarized tumor-associated macrophages (TAMs) is present in the tissue, ascitic fluid and peritoneum of ovarian cancer patients. A thorough understanding of the roles of M2-TAM in the development of ovarian cancer may provide new insight into the treatment of this disease. The rapid advancement of omics techniques presents a great challenge to biologists to extract meaningful biological information from vast pools of data. In the present study, using microarray method, we identified 996 genes in SKOV3 ovarian carcinoma cells that underwent expression level changes under the influence of TAMs. Subsequently, based on the protein-protein interactions network and the differentially expressed genes, a network showing the influence of TAMs on SKOV3 cells was constructed. The resulting network was analyzed with CFinder software and four modules were found; these modules were further analyzed using DAVID software to perform functional annotations. It was found that module I was mainly related to tumorigenesis and cell cycle. Hence, 31 genes in module I were analyzed with Cytoscape software to generate a gene-function network, which revealed that four gene proteins (E2F1, RB1, CDK2 and CDK4) were functional. Based on literature review, we postulated that CDK2 and CDK4 were key players in the network. In the subsequent molecular experiments, western blot analysis and kinase activity detection demonstrated that TAMs can significantly boost the expression levels and activities of CDK2 and CDK4 in SKOV3 cells. With ${ }^{3} \mathrm{H}-\mathrm{TdR}$ incorporation and flow cytometry assay, the proliferation and
\end{abstract}

Correspondence to: Professor Jianhua Zheng, Department of Obstetrics and Gynecology, The First Affiliated Hospital of Harbin Medical University, Harbin, Heilongjiang 150001, P.R. China E-mail: prof.jh.zheng@gmail.com

Key words: ovarian cancer, tumor-associated macrophages, microarray, CDK2, CDK4 cell cycle distribution of SKOV3 cells were detected in the absence or presence of CDK2 and CDK4 inhibitors and the results confirmed that the two kinases played a key role in TAM cells enhancing SKOV3 cell proliferation by promoting G0/G1 to $\mathrm{S}$ transition. In the present study, we identified the specific changes in the gene expression profile of SKOV3 cells under the influence of TAMs and explored a method for analyzing the gene expression profile data. The results may aid in the design of subsequent molecular experiments.

\section{Introduction}

Among the gynecological malignancies, ovarian cancer ranks third in incidence but has the highest mortality rate (1). Despite considerable advancements in traditional treatments including surgery, chemotherapy and radiation therapy, patients with advanced ovarian cancer have a long-term survival rate of $<20 \%$ (2). Hence, it is crucial to explore improved treatment options for this disease.

Classically activated (M1) macrophages and alternatively activated (M2) macrophages are two major subsets of human macrophages that exert opposite effects on the inflammatory response. Tumor-associated macrophages (TAMs) as important inflammatory components mainly exhibit M2 polarization in tumor tissues. These cells can promote the development of tumors; therefore, they have become a new target for cancer treatment $(3,4)$. Studies have revealed that TAMs are present in large numbers in ovarian cancer $(5,6)$; therefore, the elucidation of M2-TAM's roles in the development of ovarian cancer may provide new insight for the treatment of ovarian cancer.

After the human genome was sequenced, the focus of genomic studies shifted from structural to functional investigations. The rapid advancement of various omics techniques has provided platforms and opportunities to explore functional genomics and has also catalyzed the exponential growth of relevant biological data (7). It remains a challenge for biologists and mathematicians to extract biologically valuable information from these massive data sets.

The present study examined the gene expression changes between SKOV3 ovarian carcinoma cells co-cultured with 
and without TAMs using a microarray. A novel method for analyzing microarray gene expression data was explored, and the results were confirmed with molecular approaches.

\section{Materials and methods}

Cell preparation. The human ovarian cancer cell line, SKOV3, and the human monocytic leukemia cell line, THP-1, were provide by the Heilongjiang Cancer Research Institute and grown in Roswell Park Memorial Institute (RPMI)-1640 medium supplemented with $10 \%$ fetal calf serum (FBS), $100 \mathrm{U} / \mathrm{ml}$ penicillin and $100 \mu \mathrm{g} / \mathrm{ml}$ streptomycin at $37^{\circ} \mathrm{C}$ and $5 \% \mathrm{CO}_{2}$ in a humidified chamber.

To generate M2-polarized THP-1 macrophages, $1 \times 10^{6}$ THP- 1 cells were seeded into the upper insert of a 6-well Transwell system $(0.4 \mu \mathrm{M}$ pore size; Corning Life Sciences, Lowell, MA, USA) and treated with $320 \mathrm{nM}$ PMA (phorbol myristate acetate; Sigma-Aldrich, St. Louis, MO, USA) for $6 \mathrm{~h}$. They were then cultured with PMA supplemented with $20 \mathrm{ng} / \mathrm{ml}$ interleukin-4

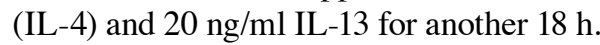

For co-culture, SKOV3 cells were plated into 6-well plates at a density of $2 \times 10^{5}$ cells/well and incubated for $24 \mathrm{~h}$. After a thorough wash to remove all PMA, M2-polarized THP-1 macrophages in the upper inserts were co-cultured with SKOV3 cells in 6-well plates. After $72 \mathrm{~h}$ of co-culture, the SKOV 3 cells were washed and used for subsequent experiments.

Detection of gene expression using a microarray. For the blank control group, cell-free culture fluids were added to the upper inserts. The SKOV3 cells were collected, and total RNA was extracted according to the instructions for the TRIzol reagent (Invitrogen, Carlsbad, CA, USA). Gene expression analyses were performed using the Human Whole Genome 8 x 60k Microarray (Agilent-027114; Genotypic Technology Pvt Ltd, Bangalore, Karnataka, India). Preparation of cRNA from $5 \mu \mathrm{g}$ of total RNA, hybridizations, washes, and detection were conducted in accordance with the protocol (http://www. genotypic.co.in/custom_micro_design.html?mnu=1). The resulting slides were scanned using the Axon GenePix 4000B microarray scanner.

Microarray data analysis. The scanned images (TIFF format) were imported into NimbleScan software (version 2.5) for grid alignment and expression data analysis. The expression data were normalized through quantile normalization and the Robust Multichip Average (RMA) algorithm included in the software. The Probe level (*_norm_RMA.pair) files and Gene level (*_RMA.calls) files were generated after normalization. All gene level files were imported into Agilent GeneSpring GX software (version 11.5.1) for further analysis. Differentially expressed genes were identified by the Student's t-test.

Bioinformatic analysis. The protein-protein interaction (PPI) network was obtained from the Human Protein Reference Database (HPRD, http://www.hprd.org) which includes 9,453 nodes and 36,874 edges (8). The differentially expressed genes were mapped onto the PPI network. The protein pairs that were interactive and both encoded by differentially expressed gene, were retained. Then the PPI network showing the influence of
TAMs on SKOV3 cells was obtained. The resulting network was analyzed with CFinder software (http://www.cfinder.org/) to identify modules (9) and each module was functionally annotated by the DAVID software (http://david.abcc.ncifcrf. gov/) (10). Specifically, enrichment analyses of KEGG pathways (Kyoto Encyclopedia of Genes and Genomes, http://www. kegg.jp) and GO-BP terms (gene oncology-biological process, http://www.geneontology.org/) for genes in each module, were performed. Then, the enriched KEGG pathways and GO-BP terms were arranged in ascending order of P-value, and the functions of the top ten KEGG pathways and GO-BP terms were examined. Those modules that were functionally interesting to us were further analyzed.

For each target module, the genes-function (the KEGG pathways or GO-BP terms) visualization networks were constructed using Cytoscape software (version 2.6.3) (11). The genes that potentially played key roles in the network were identified and analyzed.

Western blot analysis. Cells lysates were prepared with RIPA lysis buffer $(50 \mathrm{mM}$ Tris- $\mathrm{HCl}, \mathrm{pH} 7.5,50 \mathrm{mM} \mathrm{NaCl}, 1 \mu \mathrm{M}$ EGTA, $1 \%$ Triton X-100, $50 \mathrm{mM} \mathrm{NaF}, 5 \mathrm{mM} \mathrm{Na}_{3} \mathrm{VO}_{4}, 10 \mathrm{mM}$ $\mathrm{Na}_{4} \mathrm{P}_{2} \mathrm{O}_{7}, 0.1 \mathrm{mM}$ phenylmethylsulfonyl fluoride, $1 \mu \mathrm{g} / \mathrm{ml}$ aprotinin, $1 \mu \mathrm{g} / \mathrm{ml}$ pepstatin A, $1 \mu \mathrm{g} / \mathrm{ml}$ leupeptin and $1 \mathrm{mM}$ DTT) and cleared of cellular debris by centrifugation. Protein extracts were normalized for concentration by the Bio-Rad Protein Assay (Bio-Rad Laboratories, Hercules, CA, USA) and $20 \mu \mathrm{g}$ of total cell protein per sample were subjected to sodium dodecyl sulfate-polyacrylamide gel electrophoresis (SDS-PAGE) and then transferred to PVDF membranes (Amersham Inc., Arlington Heights, IL, USA). Membranes were blocked with 5\% non-fat dry milk in TBST buffer $(20 \mathrm{mM}$ Tris-HCl, pH 7.4, $150 \mathrm{mM} \mathrm{NaCl}, 0.1 \%$ Tween-20) and probed with one of the following antibodies: anti-CDK2, anti-CDK4 and anti- $\beta$-actin (Santa Cruz Biotechnology, Santa Cruz, CA, USA). Following incubation with horseradish peroxidase conjugated secondary antibody (Santa Cruz Biotechnology), the blots were visualized by ECL kit (Amersham).

CDK2 and CDK4 kinase assays. Cells were washed twice with ice-cold PBS and lysed in RIPA lysis buffer. After clearing of cellular debris by centrifugation, $500 \mu \mathrm{g}$ of cell lysate from each sample was incubated with anti-CDK2 or anti-CDK4 antibody (Santa Cruz Biotechnology) at $4^{\circ} \mathrm{C}$ overnight. Immunocomplexes were captured by Protein A-Sepharose beads (Pharmacia Biotech, Piscataway, NJ, USA) at $4^{\circ} \mathrm{C}$ for $2 \mathrm{~h}$, rocking. The precipitates were washed three times with RIPA lysis buffer and twice with kinase assay buffer (50 mM HEPES, pH 7.5, $10 \mathrm{mM} \mathrm{MgCl}_{2}, 1 \mathrm{mM}$ DTT, $10 \mathrm{mM} \beta$-glycerophosphate, $1 \mathrm{mM} \mathrm{NaF}$ and $0.1 \mathrm{mM} \mathrm{Na}_{3} \mathrm{VO}_{4}$ ), then resuspended in $30 \mu \mathrm{l}$ kinase buffer containing $100 \mu \mathrm{g} / \mathrm{ml}$ histone H1 (for CDK2 kinase assays) or $2 \mu \mathrm{g}$ of glutathione $\mathrm{S}$-transferase - $\mathrm{Rb}$ fusion protein (for CDK4 kinase assays), $50 \mu \mathrm{M}$ ATP, $0.2 \mu \mathrm{Ci} / \mu 1\left[\gamma^{-32} \mathrm{P}\right]$ ATP (Amersham Life Science) and incubated at $30^{\circ} \mathrm{C}$ for $30 \mathrm{~min}$. The reaction was stopped by adding $5 \mu \mathrm{l}$ 6x Laemmli sample buffer and boiling for $5 \mathrm{~min}$, and resolved on $10 \%$ SDS-PAGE. The gel was dried and exposed to an X-ray film. The $\left[{ }^{32} \mathrm{P}\right]-$ labeled $\mathrm{H} 1$ or Rb protein was visualized by autoradiography and the band intensities were quantified using NIH ImageJ. 


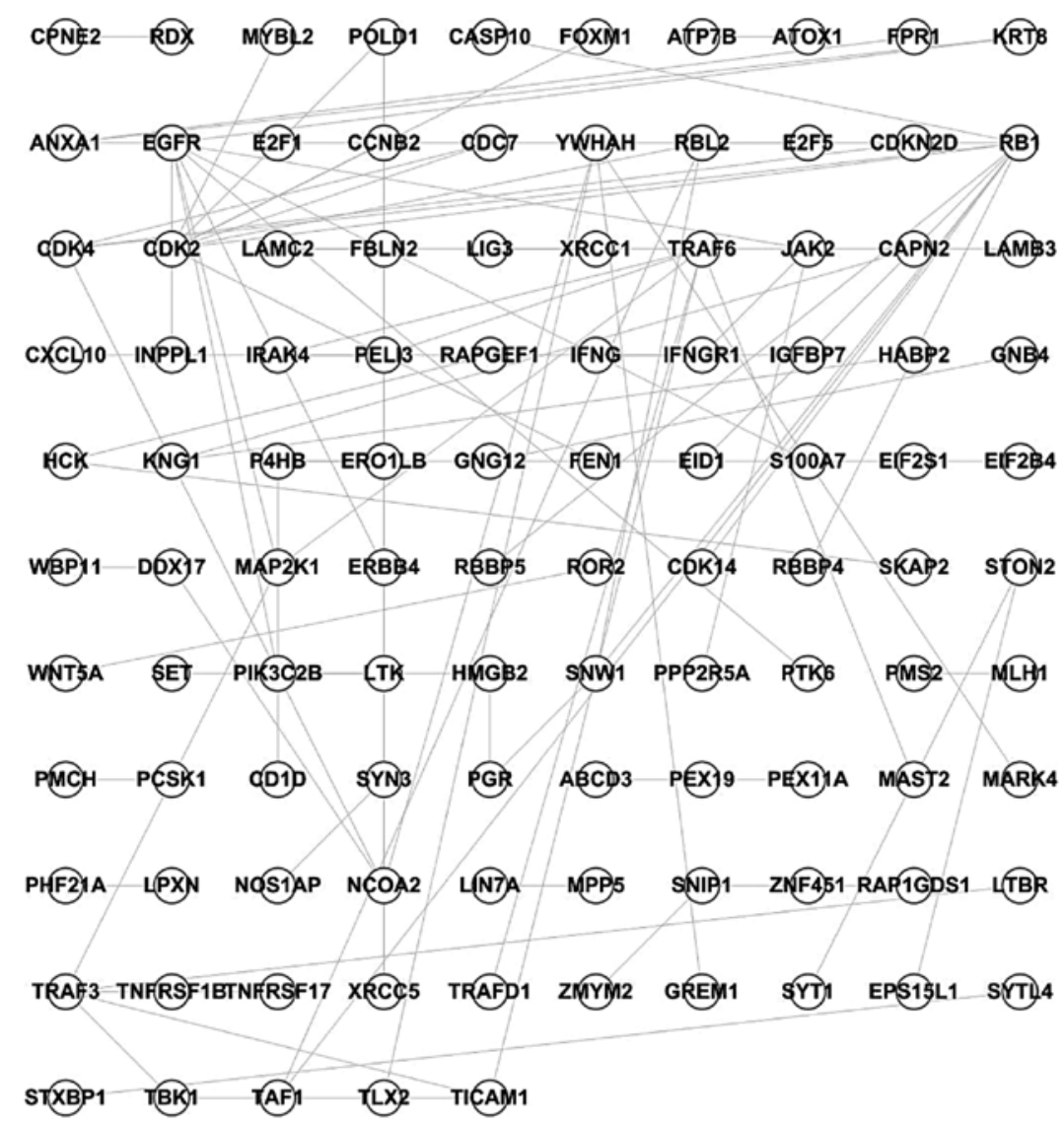

Figure 1. The network map illustrating the effects of M2-TAMs on SKOV3 ovarian carcinoma cells. SKOV3 cells were co-cultured with or without TAMs for $72 \mathrm{~h}$, and a microarray was used to examine their gene expression. The analysis yielded 996 differentially expressed genes $(\mathrm{P}<0.05)$, which were mapped onto a PPI (protein-protein interactions) network (containing 9,453 nodes and 36,874 edges). Only the protein pairs in which both proteins exhibited differential expression between the two groups were kept to generate the network, which contained 105 nodes and 91 edges. A node represents a differentially expressed gene and an edge denotes an interaction relationship between the two nodes (proteins).

${ }^{3} H$-TdR incorporation. The SKOV3 cells were seeded in 24-well plates at a density of 40,000 per well and allowed to adhere for $24 \mathrm{~h}$. After washing three times with warm PBS, the cells were subjected to growth arrest by incubation with serum-free medium for $48 \mathrm{~h}$. Then, in the presence or absence of $20 \mu \mathrm{M}$ of roscovitine (A.G. Scientific, Inc., San Diego, CA, USA) or $20 \mu \mathrm{M}$ of 3-Amino-9-thio (10H)-acridone (3-ATA; Alexis Biochemicals, San Diego, CA, USA) within the SKOV3 cell culture medium, the SKOV3 cells were cocultured with M2-polarized THP-1 macrophages in upper inserts for $72 \mathrm{~h}$. Eighteen hours before harvesting, $1 \mu \mathrm{Ci} / \mathrm{ml}$ of ${ }^{3} \mathrm{H}-\mathrm{TdR}$ was added to the medium of each well. To harvest the cells, the medium was discarded, and the cells were rapidly washed three times with PBS at $4^{\circ} \mathrm{C}$ and treated with ice-cold $10 \%$ trichloroacetic acid and neutralized with $0.2 \mathrm{M} \mathrm{NaOH}(0.5 \mathrm{ml} /$ well). The resulting lysates were transferred to scintillation vials, and thymidine incorporation was determined by scintillation counting. In each experiment, the results from triplicate wells were averaged before statistical analysis.

Cell cycle analysis. In the presence or absence of $20 \mu \mathrm{M}$ of roscovitine or $20 \mu \mathrm{M}$ of 3-ATA in the SKOV3 cell culture medium, the SKOV3 cells were co-cultured with or without M2-polarized THP-1 macrophages in the upper inserts for $72 \mathrm{~h}$. Then, the cells were harvested in cold PBS, fixed in $5 \mathrm{ml}$ of cold $70 \%$ ethanol and stored at $4^{\circ} \mathrm{C}$ for subsequent cell cycle analysis. Fixed cells were washed with PBS once and suspended in $1 \mathrm{ml}$ of propidium iodide (PI; Sigma) staining reagent $(20 \mathrm{mg} / \mathrm{ml}$ ribonuclease and $5 \mathrm{mg} / \mathrm{ml}$ of PI). The samples were incubated at $37^{\circ} \mathrm{C}$ in the dark for $30 \mathrm{~min}$ before cell cycle analysis. The distribution of cells in the cell cycle was determined by a FACSCalibur flow cytometer (BD, Biosciences, San Jose, CA, USA).

Statistical analysis. All data represent the results of three independent experiments. The data are expressed as the mean \pm SD. Significance was calculated using the Student's t-test. P-values $<0.05$ were considered to indicate statistically significant differences.

\section{Results}

$C D K 2$ and $C D K 4$ are identified for further investigation after modular analysis of the network showing the influence of TAMs on SKOV3 cells. There were a total of 996 genes that were differentially expressed between the two groups (SKOV3 vs. TAM-SKOV3 co-culture), including 653 upregulated genes and 343 downregulated genes in the co-culture group.

Using the PPI network and the 996 differentially expressed genes, a network describing the influence of TAMs on SKOV3 cells was constructed, comprised of 105 nodes and 91 edges (Fig. 1). 
A

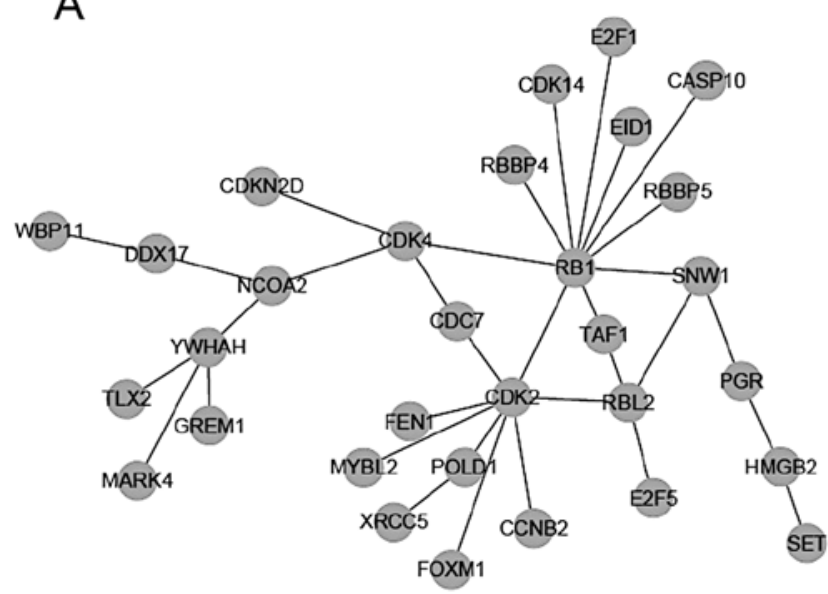

C

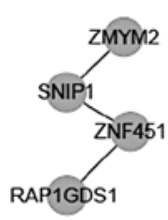

B

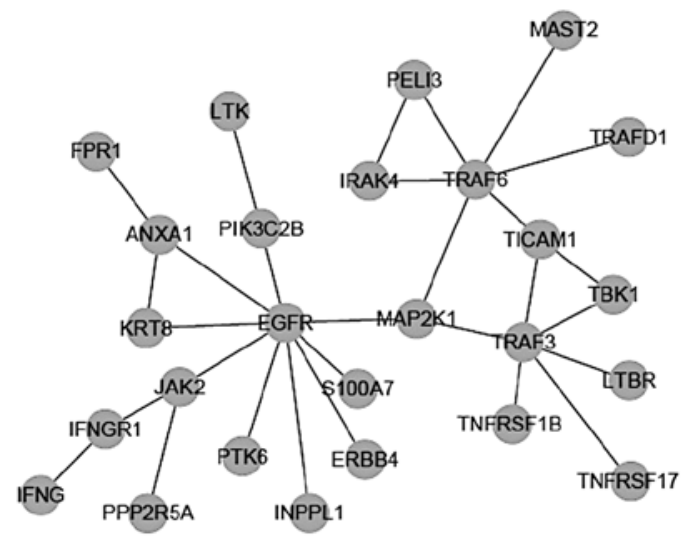

D

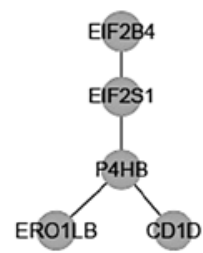

Figure 2. The modular dividing results of the network showing the influence of TAMs on SKOV3 cells. The network showing the influence of TAMs on SKOV3 cells was analyzed with CFinder software to reveal four modules. A node represents a differentially expressed gene and an edge denotes an interaction relationship between the two nodes (proteins).

Module dividing was then performed for the network, and four modules were recognized when $\mathrm{k}=3$ (Fig. 2). Via enrichment analyses of KEGG pathways and GO-BP terms for genes in each module, it was found that multiple KEGG pathways and GO-BP terms were significantly enriched for genes in modules I or II (Table I). Based on the P-value, the top ten KEGG pathways and GO-BP terms for each module were functionally examined. The genes in module I were mainly related to 'tumorigenesis' (Fig. 3A) and 'cell cycle' (Fig. 3B); thus, this module was considered a target of further analysis.

After the construction of the gene-function visualization networks (Fig. 3C and D), it was discovered that among the 31 genes in module I, 4 genes (E2F1, RB1, CDK2 and CDK4) were functional and involved in multiple cancer pathways and the 'cell cycle' pathway, as well as multiple GO-BP terms related with cell cycle.

The 4 proteins were then mapped onto a KEGG pathway termed 'pathways in cancer', in which their locations and effects were thoroughly analyzed (Fig. 4A). 'Pathways in cancer' includes 9 aspects of functional abnormalities (detailed in the 9 bigger solid boxes) in cancer. Markedly, all 4 proteins were concentrated in the 'cell-cycle' pathway and dysfunction of 'cell-cycle' pathway may lead to uncontrolled growth of tumor cells.

Subsequently, E2F1, RB1, CDK2 and CDK4 were analyzed by mapping them onto the KEGG pathways of 'cell cycle' (Fig. 4B). The 4 proteins mainly played roles in promoting cells past the G1/S checkpoint. This checkpoint has the following regulatory process: when the cell is stimulated by external signals such as growth factors, cyclin D is synthesized and binds to CDK4/CDK6 to form a complex, thereby acti-
Table I. Numbers of enriched KEGG pathways and GO-BP terms for the four modules.

\begin{tabular}{lrc}
\hline Module & GO-BP terms (n) & KEGG pathways (n) \\
\hline 1 & 70 & 13 \\
2 & 130 & 4 \\
3 & 1 & 0 \\
4 & 4 & 0 \\
\hline
\end{tabular}

vating the kinase activity of CDK4/6 (12). Activated CDK4/6 catalyzes the phosphorylation of $\mathrm{Rb}$ protein (retinoblastoma protein), thereby releasing the transcription factor E2F that otherwise binds to $\mathrm{Rb}$ (13). The free E2F enters the nucleus and induces the expression of cyclin E and CDK2 to form a cyclin E-CDK2 complex, which further boosts Rb phosphorylation and E2F releases. Then, E2F enters the nucleus and initiates the expression of a series of genes associated with DNA synthesis (14). As a consequence, DNA synthesis is elevated and drives cells to pass the G1/S checkpoint (15). The reported cell cycle regulators can be divided into three groups: cyclins, cyclin-dependent kinases (CDKs) and cyclindependent kinase inhibitors (CKIs) (16). CDKs function in the central steps of cell cycle regulation and play crucial roles in modulating cell proliferation (17). Overactive CDKs often result in uncontrolled growth of malignant tumors. Therefore, suppressing CDKs has become a new focus of tumor studies (18). 

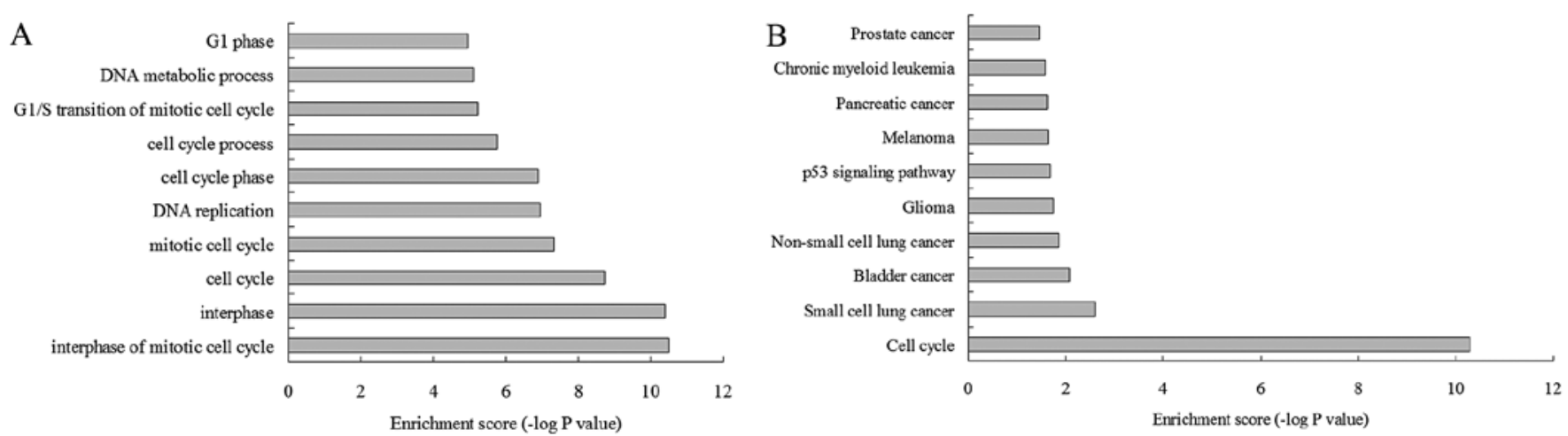

$\mathrm{C}$

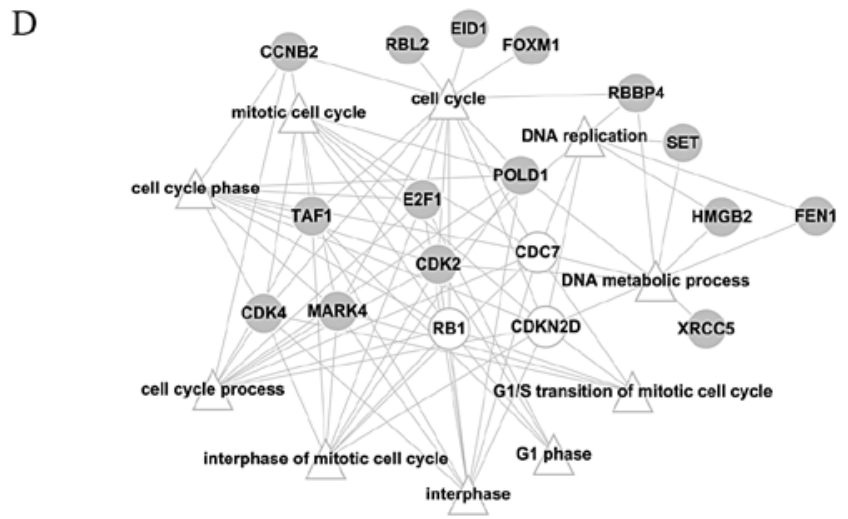

Figure 3. Results of the KEGG and GO-BP term enrichment analyses for module I. The enriched KEGG pathways and GO-BP terms for module I were arranged in ascending order of P-value $(\mathrm{P}<0.05)$. The top ten KEGG pathways (A) and GO-BP terms (B) were used to generate the bar graphs. Subsequently, the top ten KEGG pathways (C) and GO-BP terms (D), along with their corresponding proteins in module I, were used to generate a visualization network. A circular node represents a protein in module I; a hollow circle indicates that the level of the protein was lower in the TAM-SKOV3 co-culture group than in the SKOV3 group, whereas a solid circle denotes a higher protein level in the co-culture group; a triangle node stands for a KEGG pathway (C) or a GO-BP term (D). An edge connecting a KEGG pathway (C) or GO-BP term (D) to a protein indicates that the KEGG pathway (C) or GO-BP term (D) contains with the protein.

Based on the aforementioned theory and reasoning, we postulated that CDK2 and CDK4 play important roles during the process that TAMs promote SKOV3 cell proliferation. Thus, these two genes merit further study.

TAMs significantly boost the expression levels and activities of CDK2 and CDK4 in SKOV3 cells. Via western blot analysis, it was discovered that SKOV3 cells co-cultured with TAMs exhibited markedly higher CDK2 levels than their singlecultured counterparts. The CDK4 level was also higher in co-cultured SKOV3, but the difference between the two groups was not significant $(\mathrm{P}>0.05)$ (Fig. 5A). A subsequent examination of the kinase activities of CDK2 and CDK4 revealed that both kinases showed significantly higher activities in SKOV3 cells co-cultured with TAMs $(\mathrm{P}<0.01)$ (Fig. 5B). These results indicated that TAMs can enhance the expression levels and activities of CDK2 and CDK4.

Inhibitors of CDK2 and CDK4 kinases antagonize the proliferation-promoting effects of TAMs on SKOV3 cells through cell cycle arrest. Using roscovitine, a CDK2-specific inhibitor $(19,20)$ and 3-ATA, a CDK4-specific inhibitor (20), we next examined how the increased abundance and activities of CDK2 and CDK4 affect the proliferation-promoting effect of M2-TAMs on SKOV3 cells. ${ }^{3} \mathrm{H}-\mathrm{TdR}$ incorporation was first performed to investigate the proliferation of SKOV3 cells under various conditions (Fig. 6A). Roscovitine and 3-ATA reduced the proliferation rates of SKOV3 cells by 12 and $15 \%$, respectively, indicating that CDK2 and CDK4 play important regulatory roles in the normal proliferation of SKOV3 cells. After co-culture with TAMs for $72 \mathrm{~h}$, the proliferation of SKOV3 cells increased by $16 \%$. In the presence of roscovitine or 3-ATA, however, co-culture with TAM only increased the proliferation of SKOV3 cells by 4 or $2 \%$, respectively, suggesting that the two CDK inhibitors antagonize most of the proliferation-promoting effects of TAMs on the cells. These results support the idea that CDK2 and CDK4 play important roles during the process that TAMs promote SKOV3 cell proliferation.

We next utilized flow cytometry to examine the cell cycle distribution of SKOV3 cells under different conditions (Fig. 6B). Roscovitine and 3-ATA increased the number of SKOV3 cells in the G0/G1 phase and reduced the number in the $\mathrm{S}$ phase, indicating that both $\mathrm{CDK} 2$ and $\mathrm{CDK} 4$ can encourage SKOV3 cells to shift from the G1 phase to the $\mathrm{S}$ phase, thereby promoting their proliferation. After $72 \mathrm{~h}$ of co-culture with TAMs, there was a reduced proportion of SKOV3 cells in the G0/G1 phase and an increased percentage in the $\mathrm{S}$ phase, indicating that TAMs boost the transition of SKOV3 cells from the G1 phase to the S phase. In the presence of roscovitine or 3-ATA, the stimulatory effects of TAMs on the transition of SKOV3 cells from the G1 phase to the 


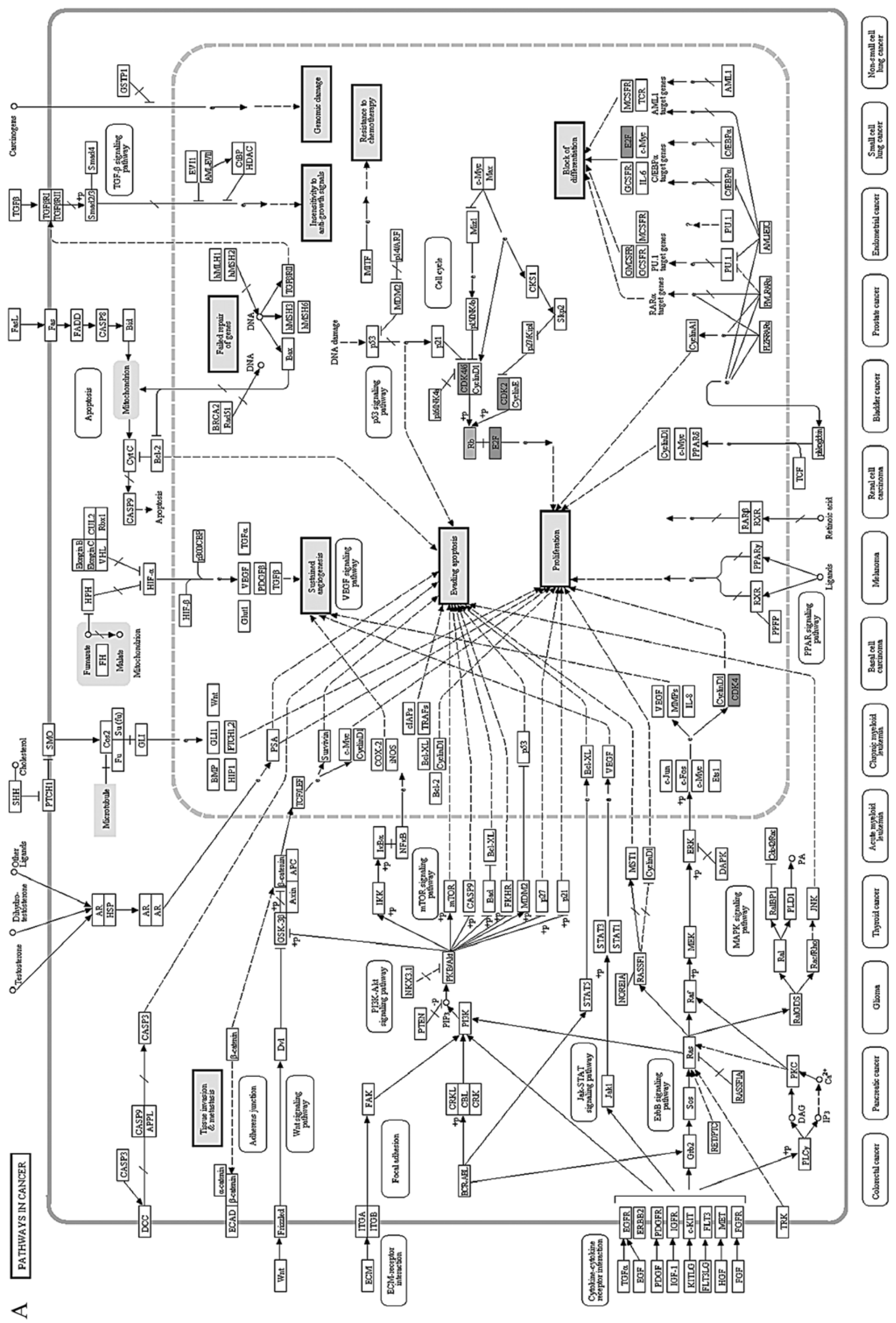




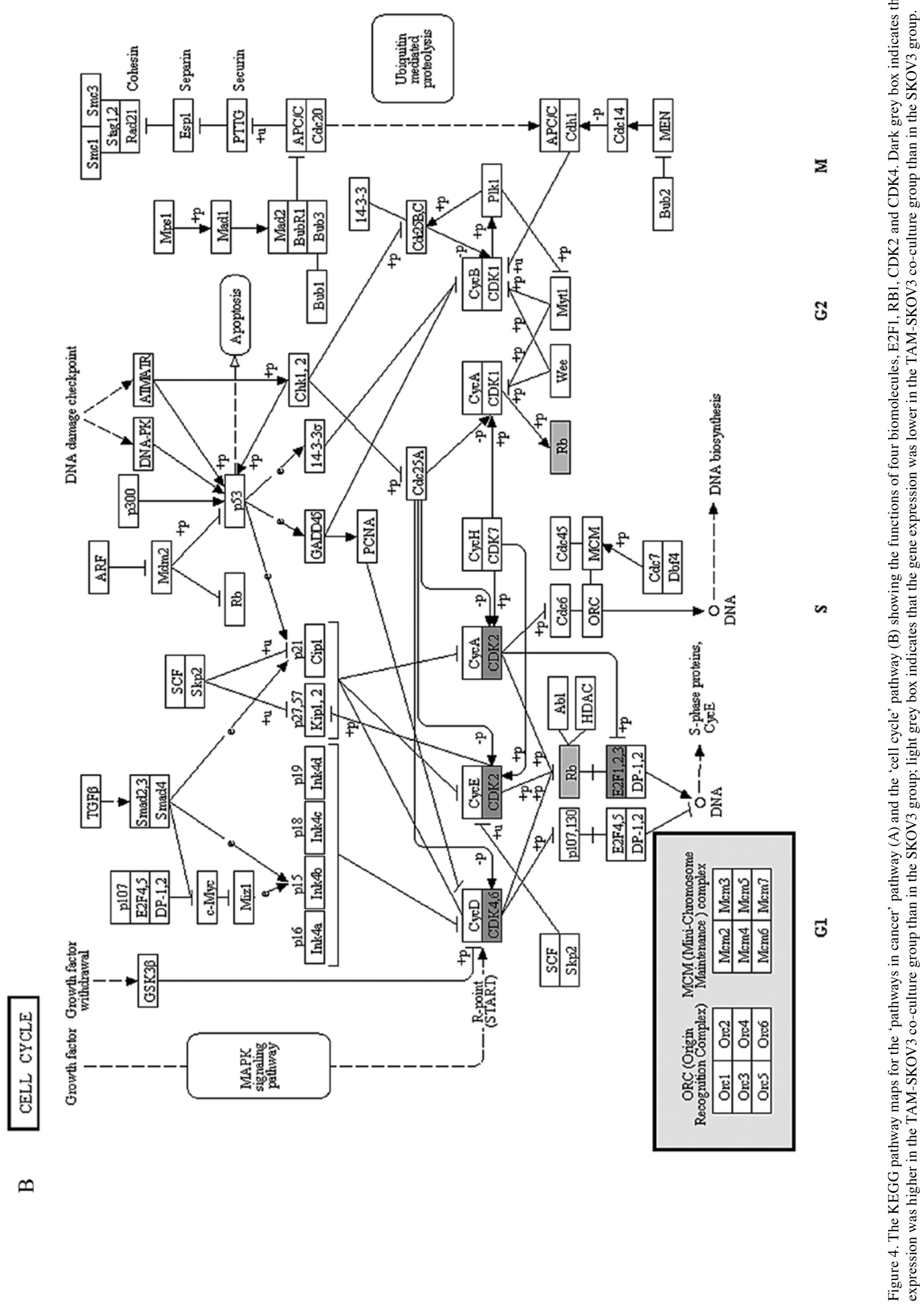


A

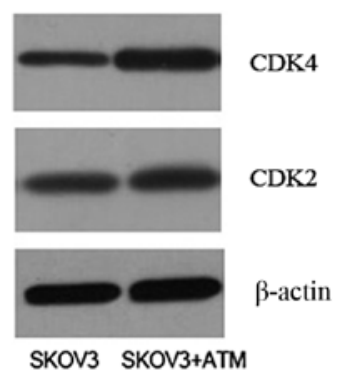

SKOV3 SKOV3+ATM

B

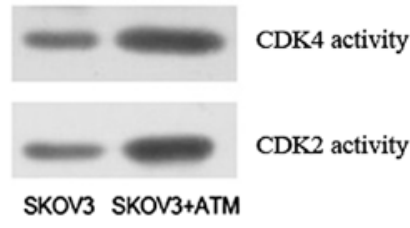

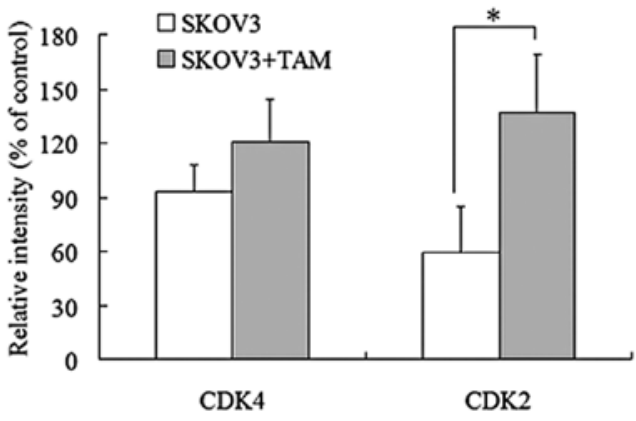

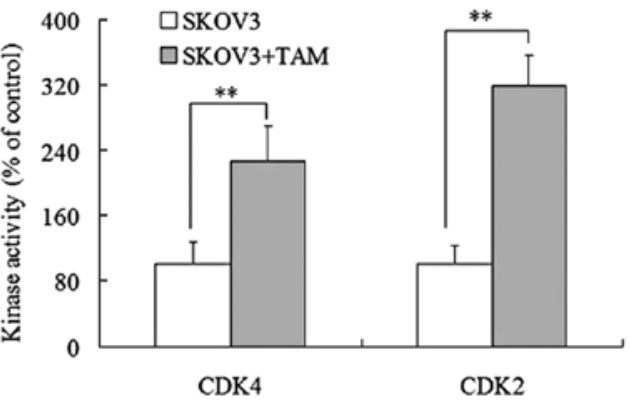

Figure 5. The effect of TAM on the expression levels and activities of CDK2 and CDK4 in the SKOV3 ovarian cancer cells. The SKOV3 cells were co-cultured with or without M2-polarized THP-1 macrophages in the upper inserts of a 6-well Transwell system for $72 \mathrm{~h}$. (A) Whole-protein extracts from the SKOV3 cells were obtained and separated by electrophoresis, and immunoblots were probed with the indicated antibodies. The housekeeping gene $\beta$-actin was used as loading control. (B) Whole protein extracts were also immunoprecipitated with the indicated antibodies and assayed for their capacity to phosphorylate histone $\mathrm{H} 1$ (for CDK2 kinase assays) or a Glutathione S-transferase-Rb fusion protein (for CDK4 kinase assays) in vitro in the presence of ${ }^{32} \mathrm{P}$ ATP. The results show the average of three separate experiments. Each column represents the mean $\pm \mathrm{SEM}$ for three independent experiments. ${ }^{*} \mathrm{P}<0.05 \mathrm{vs}$. control; ${ }^{* *} \mathrm{P}<0.01$ vs. control.

A

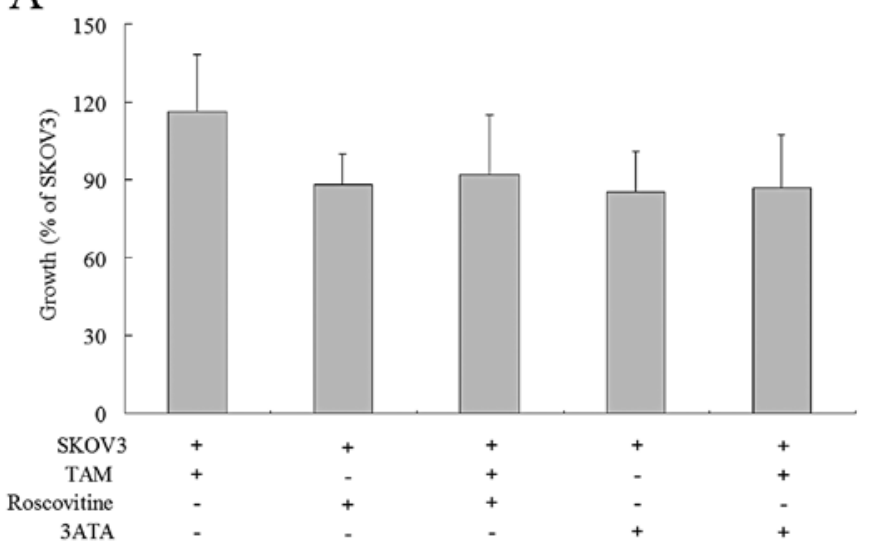

$\mathrm{B}$

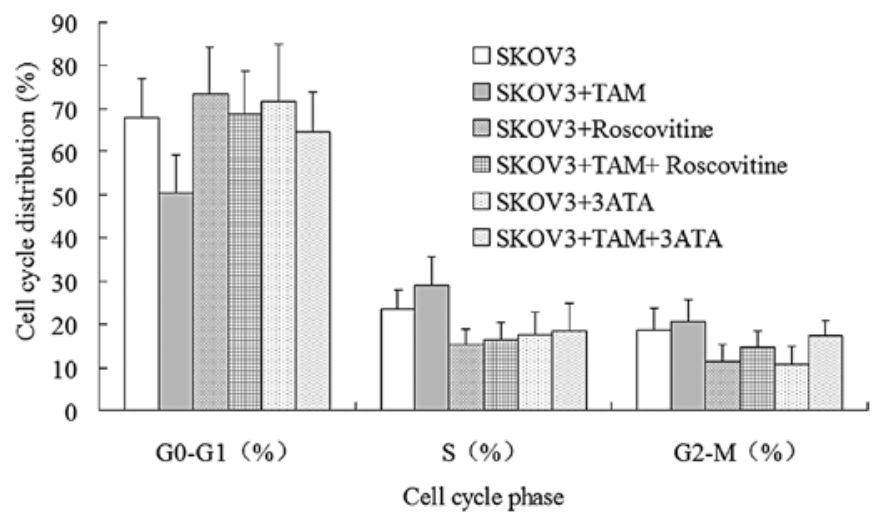

Figure 6. The effect of kinase inhibitors on growth and cell cycle progression of SKOV3 ovarian cancer cells co-cultured with TAM. (A) The SKOV3 cells were subjected to growth arrest by incubation with serum-free medium for $48 \mathrm{~h}$. Then, in the presence or absence of roscovitine or 3 -ATA within the SKOV3 cell culture medium, the SKOV3 cells were co-cultured with or without M2-polarized THP-1 macrophages in the upper inserts of a 6-well Transwell system for $72 \mathrm{~h}$. After labeling with $1 \mu \mathrm{Ci} / \mathrm{ml}$ of ${ }^{3} \mathrm{H}-\mathrm{TdR}$ for $18 \mathrm{~h}$, the SKOV3 cells were fixed with trichloroacetic acid and lysed with NaOH. Thymidine incorporation in the resulting lysates was determined by scintillation counting. (B) In the presence or absence of roscovitine or 3-ATA within the SKOV3 cell culture medium, the SKOV3 cells were co-cultured with or without M2-polarized THP-1 macrophages in the upper inserts of a 6-well Transwell system for 72 h. After propidium iodide staining, the percentage of SKOV3 cells with DNA content consistent with the Sub-G1, G0-G1, S, and G2-M phases of the cell cycle was analyzed by flow cytometry. The results are the average of three separate experiments. Each column represents mean \pm SEM for three independent experiments.

$\mathrm{S}$ phase was mostly abolished and the percentage of cells in the $\mathrm{S}$ phase was enhanced by only 1.08 and $0.82 \%$, respectively. The results indicated that CDK2 and CDK4 are crucial for the proliferation-promoting effects of TAMs on SKOV3 cells through pushing cell cycle progression from the G1 phase to the $\mathrm{S}$ phase. 


\section{Discussion}

The microenvironment of ovarian cancer consists of tumor tissues, ascitic fluid and peritoneum. Studies have demonstrated that large quantities of M2-polarized TAMs are present in these locations. Freedman et al (21) discovered that the abdominal peritonea of patients with advanced ovarian cancer were infiltrated by large quantities of mononuclear macrophages even if there was no tumor invasion. In the ascitic fluid of ovarian cancer, macrophages can account for $50 \%$ or more of the mononuclear leukocytes (22). Yip et al (23) reported that TGF- $\beta$, VEGF, IL-10, cyclooxygenase (COX) and prostaglandin E2 (PGE2) were the main immune inhibitors secreted by ovarian cancer cells and that TGF- $\beta$, VEGF, IL-10 and COX could all induce M2 polarization of TAMs. TAMs in the ovarian cancer microenvironment play a pivotal role in the progression and prognosis of the disease. Wan et al (24) reported that individuals with high-density infiltration of TAMs had a lower 5-year survival rate and that such high TAM levels is an independent prognostic factor in ovarian cancer.

In previous years, it was discovered that TAMs promote the development, invasion and metastasis of tumor cells through several mechanisms including suppressing inflammation in the acute phase, suppressing the Th1 immune response, enhancing tumor angiogenesis, inducing matrix reconstruction and improving the immune tolerance of tumor cells (25). Currently, little effort has been made to elucidate the underlying mechanisms by which TAM promotes ovarian cancer (26-28), and the relevant studies have mainly focused on the inhibition of the Th1 immune response.

Target molecules or research directions for the previous research on the mechanisms by which TAMs promote the development of ovarian cancer were mostly determined by reference to available knowledge and experience. For example, the mechanisms by which TAMs augment other types of tumors can be referenced. In fact, such experimental design is, to some degree, subjective, one-sided and difficult to grasp key issues. This is also a major disadvantage of experimental design in molecular biology. To effectively overcome this problem, the present study employed a method of systems biology in which a microarray technology was used to acquire the whole-genome differential gene expression data between SKOV3 ovarian carcinoma cells co-cultured with and without TAMs (revealing a total of 996 differentially expressed genes).

Despite the large number of new bioinformatic methods, different methods often generate enormously different results from the same set of biological data. Therefore, currently, there is no research method or model that is universally accepted. Since each analytical method has its pros and cons, it is often very difficult to choose a more reasonable method for analyzing a particular type of data. In the next step of data analysis, the present study attempted a data processing method and achieved a satisfactory outcome, as detailed below.

Specifically, we first used the differential gene expression data and the human PPI network to establish a network showing the influence of TAMs on SKOV3 cells that was composed of 105 genes. Subsequently, the network was analyzed with bionetwork-processing software for module dividing based on the modular properties intrinsic to biological networks $(29,30)$.
The analysis yielded four functional modules. To a certain degree, modules in a biological network represent functional units in life processes, such that the functional similarity between biomolecules within a module is far greater than that between those from different modules (31). Hence, dividing a biological network into modules, rather than characterizing the entire sophisticated biological network, is not only beneficial for understanding the mechanism underlying a biological function, but it also considerably reduces the complexity of the question.

After obtaining the functional modules of the network, the next step is to perform focused research. Here, we performed functional annotations using KEGG pathways and GO-BP terms for the four modules and narrowed our focus to one target module, which comprised 31 genes. After constructing a gene-function network of the target module, four genes were found functional. By comprehensively reviewing the research progress associated with the functions of these four genes, CDK 2 and CDK4 were hypothesized to play key roles in the influence of TAMs on SKOV3 cells and, thus, warranted further study.

Lastly, molecular biology experiments were performed to preliminarily demonstrate that CDK2 and CDK4 play important roles in the proliferation-promoting effects of TAMs on SKOV 3 cells. To the best of our knowledge, similar studies have not been reported.

It is apparent that systems biology methods could initiate a study from a comprehensive and global perspective. The bioinformatic approach adopted in this study narrowed down targets in a stepwise fashion and finally accurately located the crux of the problem. Therefore, the bioinformatic results can be used to effectively guide the design of molecular biology experiments, which to a certain degree can avoid being subjective and one-sided, thereby saving considerable effort, materials and money.

In summary, the present study identified the specific changes in the gene expression profile of SKOV3 ovarian carcinoma cells under the influence of TAMs and explored a method for analyzing the gene expression profile data. The results may benefit the design of subsequent molecular experiments.

\section{References}

1. Yin F, Liu X, Li D, Wang Q,Zhang W and Li L: Tumor suppressor genes associated with drug resistance in ovarian cancer (Review). Oncol Rep 30: 3-10, 2013.

2. Sorbe B, Graflund M, Nygren L, Horvath G, Swahn M, Boman K, Bangshöj R, Lood M and Malmström H: A phase II study of docetaxel weekly in combination with carboplatin every three weeks as first line chemotherapy in stage IIB-IV epithelial ovarian cancer: Neurological toxicity and quality-of-life evaluation. Int J Oncol 40: 773-781, 2012.

3. Pello OM, De Pizzol M, Mirolo M, Soucek L, Zammataro L, Amabile A, Doni A, Nebuloni M, Swigart LB, Evan GI, Mantovani A and Locati M: Role of c-MYC in alternative activation of human macrophages and tumor-associated macrophage biology. Blood 119: 411-421, 2012.

4. Okada M, Saio M, Kito Y, Ohe N, Yano H, Yoshimura S, Iwama $\mathrm{T}$ and Takami T: Tumor-associated macrophage/microglia infiltration in human gliomas is correlated with MCP-3, but not MCP-1. Int J Oncol 34: 1621-1627, 2009.

5. Zhang T, Ma Z, Wang R, Wang Y, Wang S, Cheng Z, Xu H, Jin $\mathrm{X}, \mathrm{Li} \mathrm{W}$ and Wang $\mathrm{X}$ : Thrombin facilitates invasion of ovarian cancer along peritoneum by inducing monocyte differentiation toward tumor-associated macrophage-like cells. Cancer Immunol Immunother 59: 1097-1108, 2010. 
6. Wang X, Deavers M, Patenia R, Bassett RL Jr, Mueller P, Ma Q, Wang E and Freedman RS: Monocyte/macrophage and T-cell infiltrates in peritoneum of patients with ovarian cancer or benign pelvic disease. J Transl Med 4: 30, 2006.

7. Webb S: A decade after the genome, bioinformatics comes of age. Biotechniques 51: 157-161, 2011.

8. Keshava Prasad TS, Goel R, Kandasamy K, Keerthikumar S, Kumar S, Mathivanan S, Telikicherla D, Raju R, Shafreen B, Venugopal A, Balakrishnan L, Marimuthu A, Banerjee S, Somanathan DS, Sebastian A, Rani S, Ray S, Harrys Kishore CJ, Kanth S, Ahmed M, Kashyap MK, Mohmood R, Ramachandra YL, Krishna V, Rahiman BA, Mohan S, Ranganathan P, Ramabadran S, Chaerkady R and Pandey A: Human Protein Reference Database - 2009 update. Nucleic Acids Res 37 (Database issue): D767-D772, 2009.

9. Adamcsek B, Palla G, Farkas IJ, Derényi I and Vicsek T: CFinder: locating cliques and overlapping modules in biological networks. Bioinformatics 22: 1021-1023, 2006.

10. Gao Y, Lu XC, Yang HY, Liu XF, Cao J and Fan L: The molecular mechanism of the anticancer effect of atorvastatin: DNA microarray and bioinformatic analyses. Int J Mol Med 30: 765-774, 2012.

11. Smoot ME, Ono K, Ruscheinski J, Wang PL and Ideker T: Cytoscape 2.8: new features for data integration and network visualization. Bioinformatics 27: 431-432, 2011.

12. Bretz J, Garcia J, Huang X, Kang L, Zhang Y, Toellner KM and Chen-Kiang S: Noxa mediates p18INK4c cell-cycle control of homeostasis in B cells and plasma cell precursors. Blood 117: 2179-2188, 2011.

13. Dickson MA, Tap WD, Keohan ML, D'Angelo SP, Gounder MM, Antonescu CR, Landa J, Qin LX, Rathbone DD, Condy MM, Ustoyev Y, Crago AM, Singer S and Schwartz GK: Phase II trial of the CDK4 inhibitor PD0332991 in patients with advanced CDK4-amplified well-differentiated or dedifferentiated liposarcoma. J Clin Oncol 31: 2024-2028, 2013.

14. Kolupaeva V and Basilico C: Overexpression of cyclin E/CDK2 complexes overcomes FGF-induced cell cycle arrest in the presence of hypophosphorylated $\mathrm{Rb}$ proteins. Cell Cycle 11: 2557-2566, 2012

15. Baydoun HH, Pancewicz J, Bai X and Nicot C: HTLV-I p30 inhibits multiple $\mathrm{S}$ phase entry checkpoints, decreases cyclin E-CDK2 interactions and delays cell cycle progression. Mol Cancer 9: 302, 2010.

16. Lim S and Kaldis P: Cdks, cyclins and CKIs: roles beyond cell cycle regulation. Development 140: 3079-3093, 2013.

17. Lin WR, Lai MW and Yeh CT: Cyclin-dependent kinase-associated protein phosphatase is overexpressed in alcohol-related hepatocellular carcinoma and influences xenograft tumor growth. Oncol Rep 29: 903-910, 2013.

18. Choi YH and Yoo YH: Taxol-induced growth arrest and apoptosis is associated with the upregulation of the Cdk inhibitor, p21WAF1/CIP1, in human breast cancer cells. Oncol Rep 28: 2163-2169, 2012.
19. Nair BC, Vallabhaneni S, Tekmal RR and Vadlamudi RK: Roscovitine confers tumor suppressive effect on therapy-resistant breast tumor cells. Breast Cancer Res 13: R80, 2011.

20. Shin JS, Hong SW, Lee SL, Kim TH, Park IC, An SK, Lee WK, Lim JS, Kim KI, Yang Y, Lee SS, Jin DH and Lee MS: Serum starvation induces G1 arrest through suppression of Skp2-CDK2 and CDK4 in SK-OV-3 cells. Int J Oncol 32: 435-439, 2008.

21. Freedman RS, Ma Q, Wang E, Gallardo ST, Gordon IO, Shin JW, Jin P, Stroncek D and Marincola FM: Migration deficit in monocyte-macrophages in human ovarian cancer. Cancer Immunol Immunother 57: 635-645, 2008.

22. Pollard JW: Tumour-educated macrophages promote tumour progression and metastasis. Nat Rev Cancer 4: 71-78, 2004.

23. Yip P, Chen TH, Seshaiah P, Stephen LL, Michael-Ballard KL, Mapes JP, Mansfield BC and Bertenshaw GP: Comprehensive serum profiling for the discovery of epithelial ovarian cancer biomarkers. PLoS One 6: e29533, 2011.

24. Novitskiy SV, Ryzhov S, Zaynagetdinov R, Goldstein AE, Huang Y, Tikhomirov OY, Blackburn MR, Biaggioni I, Carbone DP, Feoktistov I and Dikov MM: Adenosine receptors in regulation of dendritic cell differentiation and function. Blood 112: 1822-1831, 2008.

25. Mantovani A, Allavena P, Sica A and Balkwill F: Cancer-related inflammation. Nature 454: 436-444, 2008.

26. Saccani A, Schioppa T, Porta C, Biswas SK, Nebuloni M, Vago L, Bottazzi B, Colombo MP, Mantovani A and Sica A: p50 nuclear factor- $\kappa B$ overexpression in tumor-associated macrophages inhibits M1 inflammatory responses and antitumor resistance. Cancer Res 66: 11432-11440, 2006.

27. Curiel TJ, Coukos G, Zou L, Alvarez X, Cheng P, Mottram P, Evdemon-Hogan M, Conejo-Garcia JR, Zhang L, Burow $M$, Zhu Y, Wei S, Kryczek I, Daniel B, Gordon A, Myers L, Lackner A, Disis ML, Knutson KL, Chen L and Zou W: Specific recruitment of regulatory $\mathrm{T}$ cells in ovarian carcinoma fosters immune privilege and predicts reduced survival. Nat Med 10: 942-949, 2004

28. Kryczek I, Zou L, Rodriguez P, Zhu G, Wei S, Mottram P, Brumlik M, Cheng P, Curiel T, Myers L, Lackner A, Alvarez X, Ochoa A, Chen L and Zou W: B7-H4 expression identifies a novel suppressive macrophage population in human ovarian carcinoma. J Exp Med 203: 871-881, 2006.

29. Alon U: Biological networks: the tinkerer as an engineer. Science 301: 1866-1867, 2003

30. Ravasz E and Barabási AL: Hierarchical organization in complex networks. Phys Rev E Stat Nonlin Soft Matter Phys 67: 026112, 2003.

31. Hartwell LH, Hopfield JJ, Leibler S and Murray AW: From molecular to modular cell biology. Nature 402: C47-C52, 1999. 